\title{
Mesenchymal Mandibular Chondrosarcoma: A Case Report and Review of Literature
}

\author{
Abdeljalil Abouchadi, Hind Taoufik, Adil Arrob, Oussama Nacir, Issam Rharrassi \\ Department of Maxillo Facial, Stomatology and Plastic Surgery, Avicenne Military Teaching Hospital, Marrakech, Morocco \\ Email:pr_abouchadi@outlook.com
}

How to cite this paper: Abouchadi, A., Taoufik, H., Arrob, A., Nacir, O. and Rharrassi, I. (2018) Mesenchymal Mandibular Chondrosarcoma: A Case Report and Review of Literature. Open Journal of Stomatology, 8, 127-134.

https://doi.org/10.4236/ojst.2018.84012

Received: March 15, 2018

Accepted: April 24, 2018

Published: April 27, 2018

Copyright () 2018 by authors and Scientific Research Publishing Inc. This work is licensed under the Creative Commons Attribution International License (CC BY 4.0).

http://creativecommons.org/licenses/by/4.0/

\begin{abstract}
Introduction: Chondrosarcomas (CS) are malignant cartilaginous tumors of rare maxillary localization. Their evolution is generally slow in time. They have a high tendency for locoregional recurrence after surgical treatment. Their prognosis depends on their degree of differentiation and the quality of resection. The authors report a clinical case of mesenchymal mandibular chondrosarcoma (MC), which is considered to be a subtype of CS, and specify the epidemiological, diagnostic, therapeutic and evolutionary features of this tumor. Case Report: A 73-year-old woman reported in February 2018 in our department complained of a slowly enlarging mass involving the right part of the lower jaw for the last 18 months. On examination a firm multilobular painful mass was palpated with cortical expansion, occupying the right side. A CT scan with 3D reconstruction was achieved and showed osteolysis lesions and cortical thickening. The mass biopsy for histological examination found a grade I well-differentiated bone chondrosarcoma. We performed a hemi-mandibulectomy with complete resection of the tumor. The anatomopathologic examination of the resection specimen was in favour of a grade II mesenchymal chondrosarcoma. No radiotherapy was needed. The patient's follow up was uneventful. Discussion: CS grading is the key to its management. Low grade CS (grades I and II) of the facial skeleton are luckily the most common, they are best treated with a local resection using $1.5 \mathrm{~cm}$ margins for bone and soft tissue. According to published literature, CS was historically considered as a radio resistant tumor. Radiotherapy and chemotherapy were therefore mainly reserved for high-grade tumors and for non-operable lesions. However, radical local surgery appears to be the treatment of choice for low grade chondrosarcoma. Conclusion: CS's definitive diagnosis depends on appropriate radiographic evaluation along with adequate biopsy with meticulous histopathological examination and adjunctive immunohistochimical analysis. Neither chemotherapy nor radiotherapy is indicated as primary treatment. A long period follow-up should be advised as recurrence
\end{abstract}


and metastasis are very frequent.

\section{Keywords}

Chondrosarcoma, Mandible, Mesenchymal Tumor

\section{Introduction}

Chondrosarcoma (CS) is a locally aggressive condition resulting in the formation of cartilage rather than bone from the mesenchymal tumor cells. This condition mostly affects femur, humerus, pelvis, and the sacrum accounting for $10 \%$ - $12 \%$ of all malignant bone tumors [1]. In the maxillofacial region, maxilla is more commonly affected than the mandible, comprising less than $2 \%$ of all jaw tumors. Most CS of facial structures occurs after 30 years of age and the frequency increases with advancing age. There is no racial and gender predilection [2] [3] [4].

CS usually begins with a slow growing mass, with or without pain, associated with displaced and mobile teeth. The areas of occurrence are related to the position of embryonic remnants of cartilaginous tissue such as nasal septal cartilage in the anterior region of the maxilla and Meckel's cartilage in the posterior region of mandible. Approximately $90 \%$ of CS is known for their slow growth, low grade, non-metastasizing tumors. The remaining $10 \%$ demonstrate aggressive growth, local tissue invasion, and metastasis [5].

This clinical case of mesenchymal mandibular chondrosarcoma (MC) is reported to specify the epidemiological, diagnostic, therapeutic and evolutionary features of this tumor in the context of the existing literature.

The patient consented for the case to be published.

\section{Case Report}

A 73-year-old woman reported in February 2018 in our department complained of a slowly enlarging mass involving the right part of the lower jaw for the last 18 months. Our patient has been edentulous for 10 years and uses a removable prosthesis. She had the history of the appearance of a small nodule on non-painful inflammatory mucosa having only hindered the wearing of her dental prosthesis. Then she had noticed the slowly enlarging mass, which involved the right part of the lower jaw over time. On examination a firm multilobular mass was palpated with cortical expansion, occupying the right side, painful, non-haemorrhagic, reaching the intermaxillary commissure with obliteration of the buccal vestibule (Figure 1 ) and an exobuccal expression (Figure 2). A $1 \mathrm{~cm}$ painless mobile homolateral cervical adenopathy was found. The patient's general condition was acceptable.

The orthopantomogram radiograph showed localized osteolysis, interlesional calcification, cortical thickening and periosteal reaction, which was very sugges- 
tive of a chondrosarcoma (Figure 3). We then completed with a CT scan with $3 \mathrm{D}$ reconstruction providing a detailed view of the previously shown features (Figure 4).

A biopsy of the mass for histological examination concluded to a grade I well-differentiated bone chondrosarcoma.

Before any surgery, a thoracoabdominopelvic CT scan was performed to eliminate metastasis lesions, the results turned up normal.

A right hemimandibulectomy was planned with cervical lymph node dissection. We also anticipated the use of a pectoralis myocutaneous flap in case aggressive resection was needed (Figure 2). We haven't planned mandibular reconstruction as the patient age was advanced.

Under general anesthesia, naso tracheal intubation took place to prevent postoperative upper airway obstruction (Figure 2), and the tumor was exposed through apron incision over the submandibular region. A right Hemimandiblectomy was performed; there was no soft tissue extension, therefore we only removed a few parts of the overlying adherent skin (Figure 5). After complete removal of the tumor, a cervical node dissection was accomplished, hemostasis was achieved, drains were fixed, and wound was closed in three layers. The myocutaneous flap was not required since the resection was not aggressive. Postoperative recovery was uneventful. The operative specimen was referred for histopathological examination (Figure 6).

Microscopic study revealed tumoral malignant proliferation of amorphous appearance, with areas of chondroid differentiation, corresponding to a Grade II chondrosarcoma representing $40 \%$ of the tumor volume. There was also, an undifferentiated component, of small round, ovoid and spindle cells with scanty cytoplasm and hyperchromatic nuclei developing within chondromyxoid matrix (Figure 7). Both bone and soft tissue limits were healthy.

Immunohistochemical analysis showed diffuse positivity for CD99 and focal positivity for PS100 (Figure 8). We concluded a final diagnosis of MC.

The patient was regular to follow-up, no recurrences were reported.

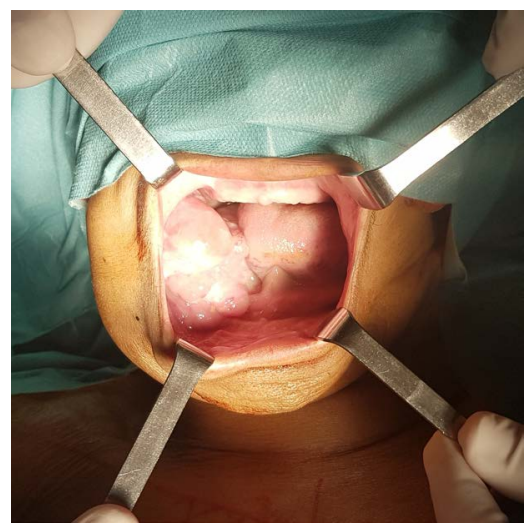

Figure 1. A mandibular multilobular non-haemorrhagic mass with cortical expansion occupying the right side. 


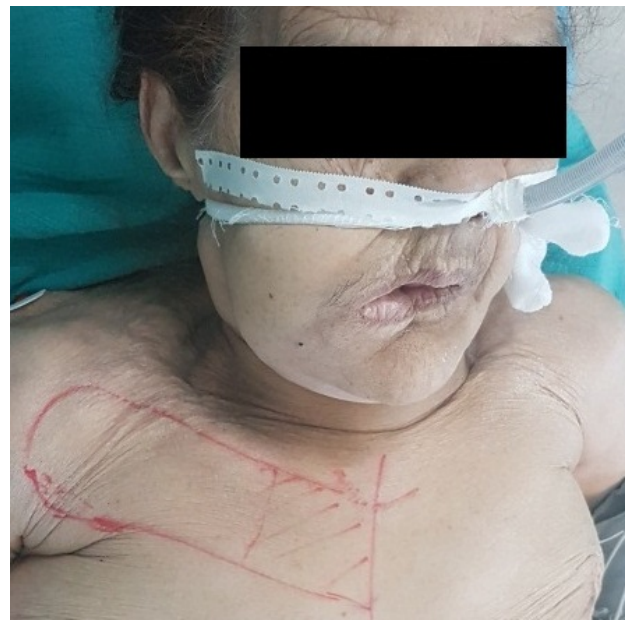

Figure 2. Patient under general anesthesia, the use of a perctoralis myocutaneous flap was anticipated.

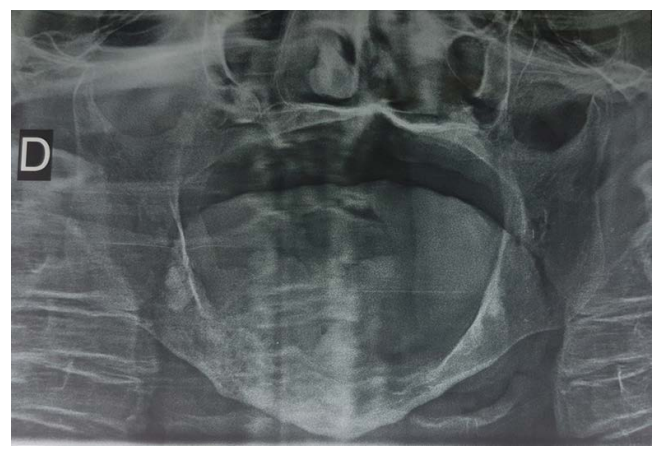

Figure 3. Localized osteolysis, interlesional calcification (popcorn calcification), cortical thickening and periosteal reaction.

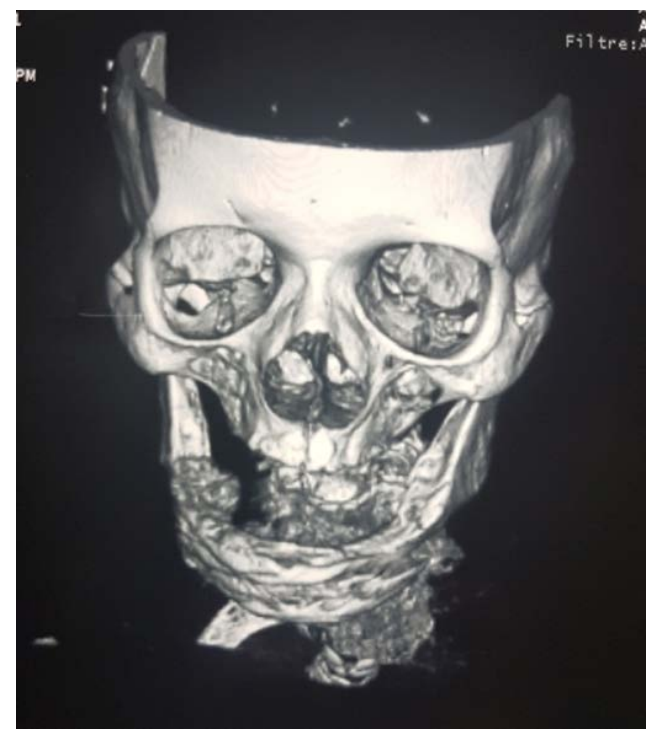

Figure 4. Scannographic 3D reconstruction images showing osteolysis. 

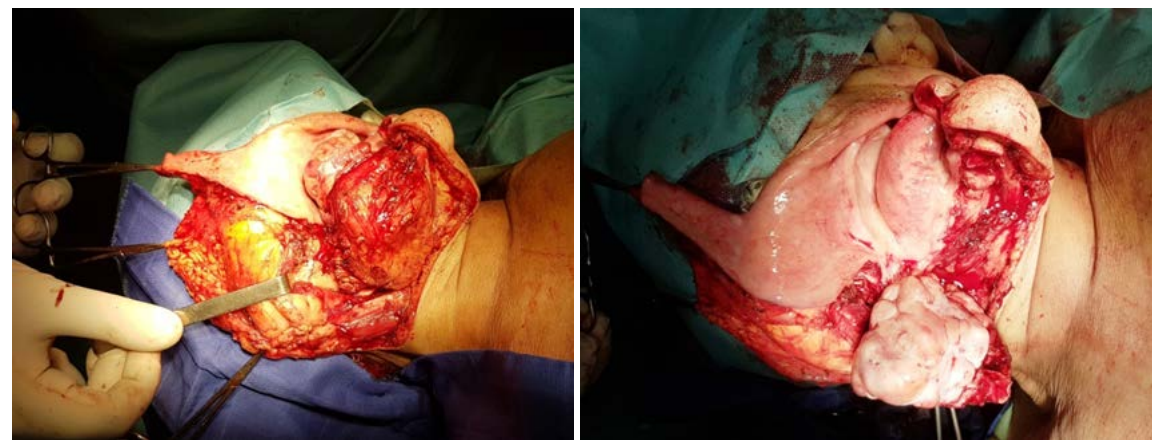

Figure 5. Exposure and extraction of the tumor through apron incision over the submandibular region.

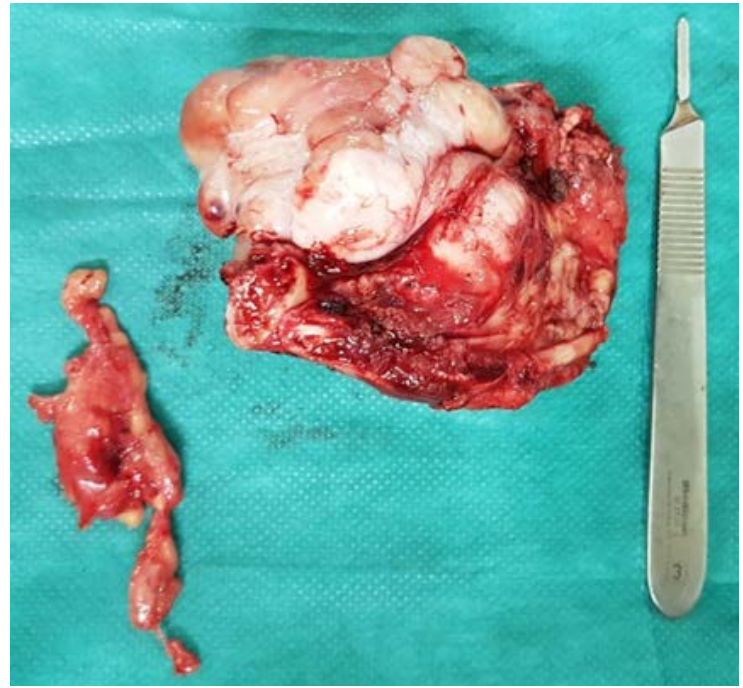

Figure 6. Operative specimen (size $9 \times 8 \times 5 \mathrm{~cm})$ : Multilobular infiltrating tumor (size $7 \times 5 \times 4.5 \mathrm{~cm}$ ) with asurrounding mucous membrane of 0.5 to $0.1 \mathrm{~cm}$.

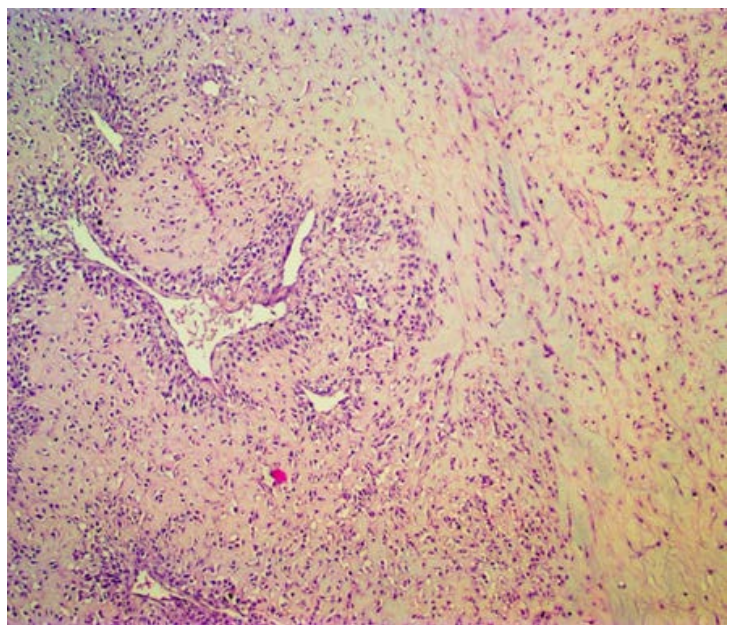

Figure 7. (View 10x) Photomicrograph of the tumor tissue showing a mesenchymal chondrosarcoma with sheets of round blue cells admixed with areas of cartilaginous differentiation. 


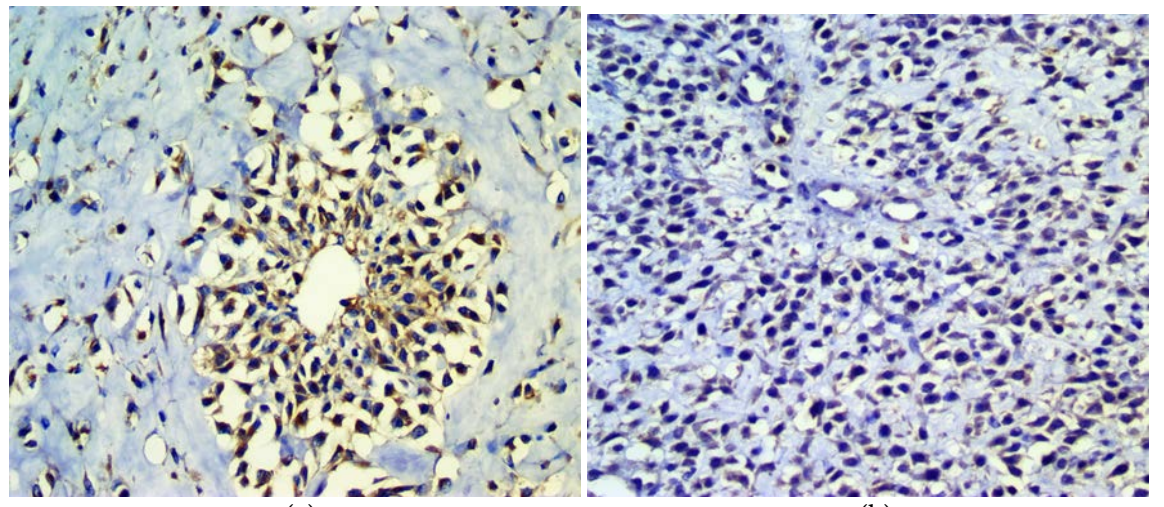

(a)

(b)

Figure 8. Immunophenotyping of chondrosarcoma cells: (a) Positive CD99 for small cell component; (b) Focal positivity for anti PS100.

\section{Discussion}

CS originates from mesenchymal stem cells and undergoes a partial differentiation to form chondroblastic differentiation and even definable cartilage. In 1942, Lichtenstein and Jaffe described CS as a lesion that develops directly from a sarcomatous stroma, developing from full-fledged cartilage and not showing neoplastic osteoid tissue and bone [6]. MC is a specific variant of CS [7] and represents approximately $1 \%$ of all CSs. [8]. It was first described by Lichtenstein $\mathrm{L}$ and Bernstein D in 1959 as a biphasic tumor, comprising of spindle cell mesenchyme interspersed with areas of chondroid differentiation. The predominant clinical symptom is painless mass or swelling. Other most commonly reported symptoms are nasal obstruction, epistaxis, tooth mobility, headache, bleeding, ulceration, facial asymmetry, paresthesia and trismus [9].

The radiographic appearance of $\mathrm{MC}$ in the jaws varies from being radiolucent to a mixed radiolucency/radiopacity. In the mandible, these lesions are generally radiolucent, with zones of radiopacity in the lytic areas [10]. Present case showed mixed radiolucency and radiopacity. Diagnosis can only be established by combination of clinical, radiographic and histopathological examination [11].

The histologic appearance of MC is characteristically biphasic pattern. Undifferentiated areas appear as sheets of primitive mesenchymal spindle/round cells similar to small cell anaplastic sarcoma. However, islands of relatively well differentiated cartilaginous tumor help in making a specific diagnosis. Calcification or ossification may occur within the chondroid matrix. Histologically, the lesion must be differentiated from similar other lesions like hemangiopericytoma, Ewing's sarcoma, PNET, leukemia/lymphoma, rhabdomyosarcoma, and malignant melanoma [12].

On scannography, it appears as irregular intramedullary radiolucency causing cortical expansion and destruction. Punctate radio opacities due to dystrophic calcifications or focal ossifications of cartilage can be seen [13]. A widening of the periodontal ligament space (Garrington's sign) may be found in the tooth bearing areas as an early sign of CS, just as it is an early sign of osteosarcoma. 
Magnetic resonance imaging (MRI) is more perceptible to cartilage and cartilaginous tumors and provides a better tracing of tumor extent than a CT scan. Even if metastasis of CS is less common than with other sarcomas, a chest radiographic exam is required to exclude this most frequent localization of metastasis [14]. In our case, athoracoabdominopelvic CT scan for this purpose.

CS grading is the key to its management. low grade CS (grades I and II) of the facial skeleton are luckily the most common, they are best treated with a local resection using $1.5 \mathrm{~cm}$ margins for bone and soft tissue. Neither chemotherapy nor radiotherapy is indicated as primary treatment. These lesions can be invasive but they are typically for their slow growth; lymph node metastasis is therefore rare, and cervical node dissection is not necessarily required [15] [16]. The uncommon high-grade CS (grade III) is treated with an initial aggressive resection of a $3 \mathrm{~cm}$ marge in the bone and $2 \mathrm{~cm}$ in soft tissues, the surgery is usually followed by chemotherapy. The prognosis for low grade CS is excellent. The 5 year survival rates are $90 \%$ for grade I and $81 \%$ for grade II. However, high grade (grade III) CS only has a $29 \% 5$ year survival rate. High grade (grade III) CS more often fails to be cured, it often evolves to distant metastasis (66\%): lungs, sternum, and vertebrae are the most common localizations. However, lymph node metastasis also can ensue if the initial therapy did not involve a cervical lymph node dissection [3] [15] [17].

In the current case, we performed a wide local excision with a tumor-free margin of 2 to $3 \mathrm{~cm}$. Neither radiotherapy nor chemotherapy was indicated. We advised a long period follow-up to which the patient is regular. She didn't present any recurrences so far.

\section{Conclusion}

Present case highlights that the diagnosis of MC should be assessed cautiously. Along with radiographic evaluation, adequate biopsy with meticulous histopathological examination of the multiple sections along with adjunctive immunohistochimical analysis is the key for the definitive diagnosis. According to published literature, extensive resection has less recurrence and a better survival rate, while radiotherapy and chemotherapy are still a controversial topic. A long period follow-up should be advised as recurrence and metastasis are very frequent.

\section{References}

[1] Burkey, B.B., Hoffman, H.T., Baker, S.R., Thornton, A.F. and McClatchey, K.D. (1990) Chondrosarcoma of the Head and Neck. Laryngoscope, 100, 1301-1305. https://doi.org/10.1288/00005537-199012000-00010

[2] Saito, K., Unni, K.K., Wollan, P.C. and Lund, B.A. (1995) Chondrosarcoma of the Jaw and Facial Bones. Cancer, 76, 1550-1558.

https://doi.org/10.1002/1097-0142(19951101)76:9<1550::AID-CNCR2820760909>3. $\underline{0 . \mathrm{CO} ; 2-\mathrm{S}}$

[3] Ruark, D.S., Schlehaider, U.K. and Shah, J.P. (1992) Chondrosarcoma of the Head 
and Neck. World Journal of Surgery, 16, 1010-1016.

https://doi.org/10.1007/BF02067021

[4] Garrington, G.E. and Collett, W.K. (1988) Chondrosarcoma. II. Chondrosarcoma of the Jaws: Analysis of 37 Cases. Journal of Oral Pathology \& Medicine, 17, 12-20. https://doi.org/10.1111/j.1600-0714.1988.tb01498.x

[5] da Rosa Santos, O.L., Moreira, A.M., Lopes Cardoso, I.C., Rodrigues Terra, B.A., Bellizzi, A. and Ramos-e-Silva, M. (1996) Sarcoma of Meckel's Cartilage. International Journal of Dermatology, 35, 353-354. https://doi.org/10.1111/j.1365-4362.1996.tb03637.x

[6] Lichtenstein, L. and Jaffe, H.L. (1943) Chondrosarcoma of Bone. American Journal of Pathology, 19, 553-589.

[7] Brown, R.E. and Boyle, J.L. (2003) Mesenchymal Chondrosarcoma: Molecular Characterization by a Proteomic Approach with Morphogenic and Therapeutic Implications. Annals of Clinical \& Laboratory Science, 33, 131-141.

[8] Cheim, A.P., Queiroz, T.L., Alencarc, W.M., Rezende, R.M. and Vencio, E.F. (2011) Mesenchymal Chondrosarcoma in the Mandible: Report of a Case with Cytological Findings. Journal of Oral Science, 53, 245-247.

https://doi.org/10.2334/josnusd.53.245

[9] Thomas, R.S., Sherlin, H.J., Natesan, A., Premkumar, P., Ramani, P., Chandrasekar, T., et al. (2010) Mesenchymal Chondrosarcoma: A Report of Two Cases with Immunohistochemical Study. Iranian Journal of Pathology, 55, 100-104.

[10] Aziz, S.R., Miremadi, A.R. and McCabe, J.C. (2002) Mesenchymal Chondrosarcoma of the Maxilla with Diffuse Metastasis: Case Report and Literature Review. Journal of Oral and Maxillofacial Surgery, 60, 931-935. https://doi.org/10.1053/joms.2002.33865

[11] Anil, S., Beena, V.T., Lal, P.M. and Varghese, B.J. (1998) Chondrosarcoma of the Maxilla. Case Report. Australian Dental Journal, 43, 172-174.

https://doi.org/10.1111/j.1834-7819.1998.tb00160.x

[12] Jaetli, V. and Gupta, S. (2011) Mesenchymal Chondrosarcoma of Maxilla: A Rare Case Report. Medicina Oral, Patologia Oral Y Cirugia Bucal, 16, e493-e496. https://doi.org/10.4317/medoral.16.e493

[13] Takahashi, K., Sato, K., Kanazawa, H., Wang, X.L. and Kimura, T. (1993) Mesenchymal Chondrosarcoma of the Jaw-Report of a Case and Review of 41 Cases in the Literature. Head Neck, 15, 459-464. https://doi.org/10.1002/hed.2880150516

[14] Garrington, G.E. and Collett, W.K. (1988) Chondrosarcoma. II. Chondrosarcoma of the Jaws: Analysis of 37 Cases. Journal of Oral Pathology \& Medicine, 17, 12-20. https://doi.org/10.1111/j.1600-0714.1988.tb01498.x

[15] Murayama, S., Suzuki, I., Nagase, M., Shingaki, S., Kawasaki, T., Nakajima, T., et al. (1988) Chondrosarcoma of the Mandible. Report of Case and a Survey of 23 Cases in the Japanese Literature. Journal of Cranio-Maxillo-Facial Surgery, 16, 287-292. https://doi.org/10.1016/S1010-5182(88)80063-5

[16] Weiss, W.W., Jr. and Bennett, J.A. (1986) Chondrosarcoma: A Rare Tumor of the Jaws. Journal of Oral and Maxillofacial Surgery, 44, 73-79. https://doi.org/10.1016/0278-2391(86)90017-0

[17] Evans, H.L., Ayala, A.G. and Romsdahl, M.M. (1977) Prognostic Factors in Chondrosarcoma of Bone: A Clinicopathologic Analysis with Emphasis on Histologic Grading. Cancer, 40, 818-831. https://doi.org/10.1002/1097-0142(197708)40:2<818::AID-CNCR2820400234>3.0.C $\underline{\mathrm{O} ; 2-\mathrm{B}}$ 